\title{
Phase retrieval of coherent diffractive images with global optimization algorithms
}

DOI:

$10.1107 / \mathrm{S} 1600576717013012$

\section{Document Version}

Accepted author manuscript

Link to publication record in Manchester Research Explorer

\section{Citation for published version (APA):}

Truong, N. X., Whittaker, E., \& Denecke, M. A. (2017). Phase retrieval of coherent diffractive images with global optimization algorithms. Journal of Applied Crystallography, 50(6), 1637-1645.

https://doi.org/10.1107/S1600576717013012

\section{Published in:}

Journal of Applied Crystallography

\section{Citing this paper}

Please note that where the full-text provided on Manchester Research Explorer is the Author Accepted Manuscript or Proof version this may differ from the final Published version. If citing, it is advised that you check and use the publisher's definitive version.

\section{General rights}

Copyright and moral rights for the publications made accessible in the Research Explorer are retained by the authors and/or other copyright owners and it is a condition of accessing publications that users recognise and abide by the legal requirements associated with these rights.

\section{Takedown policy}

If you believe that this document breaches copyright please refer to the University of Manchester's Takedown Procedures [http://man.ac.uk/04Y6Bo] or contact uml.scholarlycommunications@manchester.ac.uk providing relevant details, so we can investigate your claim.

\section{OPEN ACCESS}




\title{
Non-perturbative Harmonic Generation in Graphene from Intense Mid-Infrared Pulsed Light
}

\author{
M. Taucer,* T. J. Hammond, P. B. Corkum, and G. Vampa \\ Joint Attosecond Science Laboratory, University of Ottawa, Ottawa, Ontario K1N 6N5, Canada. \\ C. Couture, N. Thiré, B. Schmidt, and F. Légaré \\ INRS-EMT, 1650 Boulevard Lionel-Boulet, CP 1020, Varennes, Qubec J3X 1S2, Canada. \\ H. Selvi, N. Unsuree, B. Hamilton, T. J. Echtermeyer, and M. A. Denecke \\ Photon Science Institute, University of Manchester, Manchester M13 9PL, United Kingdom.
}

\begin{abstract}
In solids, high harmonic radiation arises from the sub-cycle dynamics of electrons and holes under the action of an intense laser field. The strong field regime opens new opportunities to understand and control carrier dynamics on ultrafast time scales, including the coherent dynamics of quasiparticles such as massless Dirac fermions. Here, we irradiate monolayer and few-layer graphene with intense infrared light to produce non-perturbative harmonics of the fundamental up to the 7th order. We find that the polarization dependence shows surprising agreement with gas phase harmonics. Using a two-band model, we explore the nonlinear current due to electrons near the Dirac points, and we discuss the interplay between intraband and interband contributions to the harmonic spectrum. This interplay opens new opportunities to access ultrafast and strong-field physics of graphene.
\end{abstract}

\section{INTRODUCTION}

The interaction of intense light with atoms and molecules, mainly in the gas phase, produces high harmonics. This non-perturbative process occurs when electrons are pulled away from their host atoms or molecules in the laser field and accelerated back toward the host ion as the field reverses. If the electron and ion recombine, they emit coherent high energy photons ${ }^{1}$. The success of strong field physics for gas phase atoms and molecules has motivated a search for similar effects in the solid state. Recently, high harmonic emission has been observed from a range of solids ${ }^{2-4}$, including $\mathrm{ZnO}^{5,6}$, silicon $^{7}$, and recently transition metal dichalcogenides ${ }^{8,9}$. The observations are important because the high harmonics are not only a unique source of radiation, they are also a new way of studying material. Important for this paper, high harmonic generation in the solid state opens the possibility of studying the high field response of novel materials and confined systems of low dimensionality. Two-dimensional materials, in particular, exhibit unique electronic properties and quasiparticles, including massless Dirac fermions in graphene.

Non-linear optical properties of graphene have been an active area of research recently, with demonstrations of perturbative processes including four-wave mixing ${ }^{10}$ and third harmonic generation ${ }^{11}$. Beyond this, the nonperturbative, strong-field regime has also been the focus of theoretical and experimental work. Early studies predicted highly efficient high harmonic generation due to the motion of carriers in the unusual band structure of graphene $^{12,13}$, and subsequent theoretical work has revealed the importance of the subtle interplay between interband and intraband processes ${ }^{14-17}$. Meanwhile, there have been predictions of new effects in the strong-field regime for wavelengths from the $\mathrm{THz}$ to the optical part of the electromagnetic spectrum, including ultrafast interferometry and signatures of Berry phase ${ }^{18}$, as well as a recent prediction of plasmonic enhancement of high harmonic generation ${ }^{19}$. Experimentally, the strong-field regime has been studied in the $\mathrm{THz}$ regime, showing evidence of odd harmonics up to the fifth order ${ }^{20,21}$, in agreement with theory ${ }^{14,15,22}$.

Here, we measure the intensity and polarization dependence of non-perturbative harmonics generated in monlayer and few-layer graphene. We compare the polarization dependence with predictions of the gas phase recollision model, showing striking agreement. Using a twoband model, we consider the contributions of interband and intraband currents. While the intraband contribution is greater, we discuss the possibility of observing their coherent interplay due to an intensity-dependent phase difference. This opens a new route to experimentally accessing strong-field and ultrafast processes in graphene.

Graphene consists of a single atomic layer of carbon arranged in a honeycomb lattice. Its crystal structure, together with the $\mathrm{sp}^{2}$ bonding of its constituent atoms, leads to the unique band structure shown in Fig. 1a. The out-of-plane p-orbitals, treated here within the nearestneighbour tight-binding model, give rise to a zero band gap and linear dispersion at the $\mathrm{K}$ and $\mathrm{K}^{\prime}$ points (also known as Dirac points) in reciprocal space ${ }^{23}$, shown as different coloured stars in Fig. 1b. In the so-called Dirac cones near these high-symmetry points, electrons and holes act as massless Fermions, with a constant velocity (the Fermi velocity) of $v_{F}=\sqrt{3} a t_{0} / 2 \hbar$, where $a=2.46 \AA$ is the lattice constant, and $t_{0}=2.9 \mathrm{eV}$ is an energy describing hopping between nearest neighbours ${ }^{24}$. A small gap, less than $1 \mathrm{meV}$, is present as a result of spin-orbit 

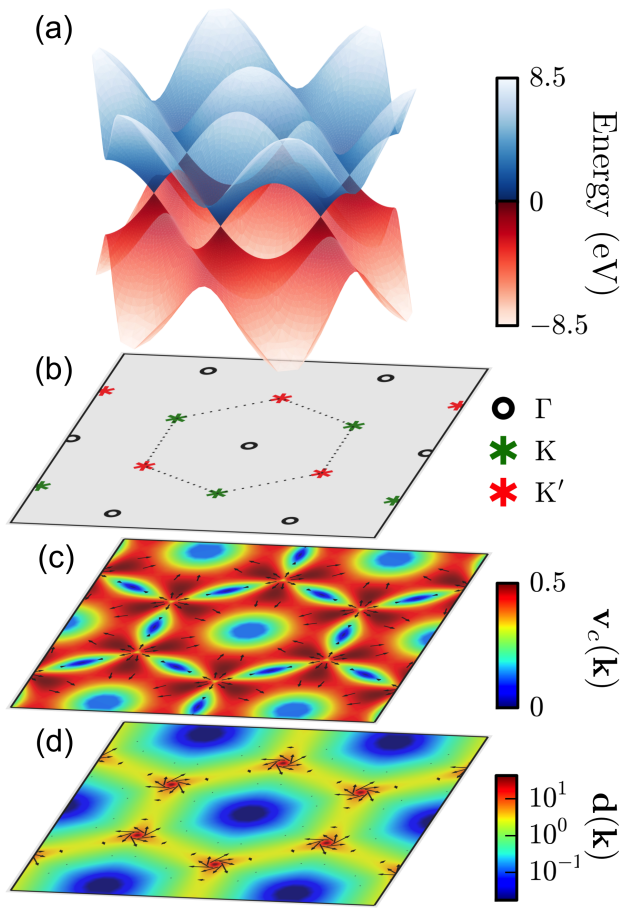

FIG. 1. (a) Band structure of graphene within the nearestneighbour tight-binding approximation. Conduction and valence bands are shown in blue and red, respectively. (b) Reciprocal space, with reciprocal lattice sites shown as circular dots, including the $\Gamma$ point at the origin. Dirac points are indicated by stars, with different colors for the inequivalent $\mathrm{K}$ and $\mathrm{K}$ ' points. The dotted line shows the boundary of the first Brillouin zone. (c) Electron velocity in the conduction band, as a function of reciprocal space coordinate. Color represents the magnitude of velocity, and arrows indicate its direction. (d) Transition dipole moment as a function of reciprocal space coordinate. Color shows the magnitude on a logarithmic scale, and the direction is indicated by arrows. Unless otherwise indicated, we use atomic units.

coupling ${ }^{25}$. Fig. 1c shows the velocity of electrons in the conduction band. Near the Dirac points, the electron velocity points radially outward with a nearly constant magnitude. The coupling between bands in the optical field is given by the transition dipole moment, shown in Fig. 1d, which diverges at the Dirac points and has opposite curls for the $\mathrm{K}$ and $\mathrm{K}^{\prime}$ points $^{26}$.

\section{EXPERIMENTAL DETAILS}

Two sets of samples were prepared for the measurements, multi- and single-layer graphene. For the multilayer samples, graphene was mechanically exfoliated from bulk graphite onto a layer of polymethyl methacrylate (PMMA) and characterized by atomic-force-microscopy yielding thicknesses in the range of $5-15 \mathrm{~nm}$. Subsequently, the graphene flakes including the PMMA support were transferred onto the sapphire target substrate $\left(\mathrm{Al}_{2} \mathrm{O}_{3}\right.$, c-plane (0001) with a thickness of $\left.500 \mu \mathrm{m}\right)$ and the PMMA removed with acetone. Single layer graphene samples were prepared from commercially sourced CVDgrown graphene on copper foil. Using PMMA as a support, the copper foil was etched away and the PMMA graphene stack transferred to the $500 \mu \mathrm{m}$ thick sapphire target substrate. The PMMA supporting layer was then removed with acetone.

Our measurements were performed on the $100 \mathrm{~Hz}$ repetition rate laser at the Advanced Laser Light Source (ALLS). We generated harmonics using femtosecond pulses with central wavelengths between 3.1 and $3.9 \mu \mathrm{m}$. The pulse duration was $70 \mathrm{fs}$, and pulse energies were between 3 and $16 \mu \mathrm{J}$. The Rayleigh length was 8-10 mm. In order to avoid damage and to increase signal during the experiments, we moved the monolayer sample four to five centimeters in front of the focus. This allowed us to increase the pulse energy for a given peak intensity by increasing the beam width at the sample. Pulse intensities were in the range of $5.7 \times 10^{10}$ to $5.5 \times 10^{11} \mathrm{~W} / \mathrm{cm}^{2}$. The latter is found to be just below the threshold for damage. All reported intensities in this article refer to vacuum intensities.

\section{RESULTS AND DISCUSSION}

Fig. 2a shows a harmonic spectrum covering the 5th and 7th order acquired from few-layer graphene with a driving wavelength of $3.6 \mu \mathrm{m}$ and an intensity of $5.5 \times 10^{11} \mathrm{~W} / \mathrm{cm}^{2}$. Fig. $2 \mathrm{~b}$ shows the harmonic spectrum near the 5th harmonic from a single layer of graphene with a driving wavelength of $3.1 \mu \mathrm{m}$ and an intensity of $5.7 \times 10^{10} \mathrm{~W} / \mathrm{cm}^{2}$. Fig. 2c shows the intensity of emitted harmonics as a function of the intensity of the incident fundamental. Solid lines show the theoretical prediction of the two band model, described in more detail below. Theoretical fits for the fifth and seventh harmonic are each scaled by an arbitrary factor. The predicted ratio between these harmonics is indicated by the vertical dashed line, which shows that our theory underestimates their difference. Theory and experiment both show non-perturbative scaling roughly characterized by a power law $\left(\propto I^{2}\right)$.

The harmonic peaks are broader in the few-layer sample compared to the monolayer, as shown in Fig. 2d, where monolayer and few-layer sample results are compared at the same driving wavelength; the full-width at half maximum of the fifth harmonic peak increases from $24 \mathrm{~nm}$ to $44 \mathrm{~nm}$. We attribute this to self-phase modulation, which causes a distortion of the driving pulse as it propagates through the few-layer graphene. The nonlinear index of few- and single-layer graphene has been estimated to be in the range of $10^{-7}$ to $10^{-9} \mathrm{~cm}^{2} / \mathrm{W}^{27-30}$. The broadening we observe is consistent with a large nonlinear index on the order of $10^{-9} \mathrm{~cm}^{2} / \mathrm{W}$ (see Appendix A).

In all cases, we observed a clear dependence on ellipticity of the incident pulse, with harmonic intensity falling 

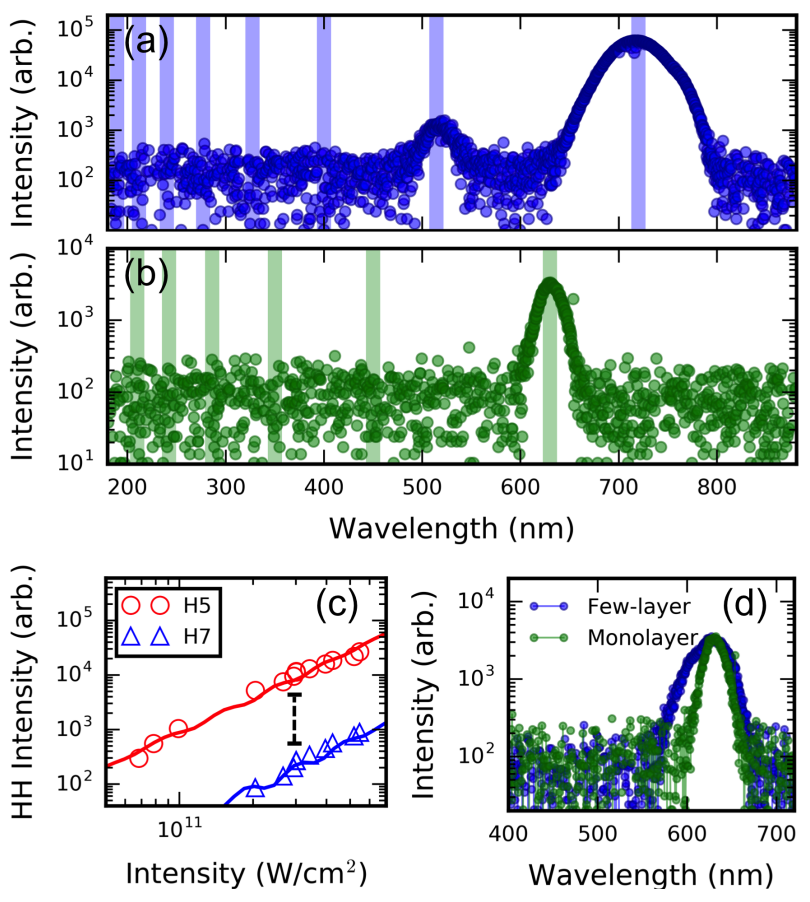

FIG. 2. (a) Harmonic spectrum from few-layer graphene with a driving wavelength of $3.6 \mu \mathrm{m}$, intensity of $5.5 \times 10^{11} \mathrm{~W} / \mathrm{cm}^{2}$, and beam diameter of $100 \mu \mathrm{m}$. The ratio of the 5 th harmonic intensity to the 7th is approximately 50. (b) Harmonic spectrum from single-layer graphene with a driving wavelength of $3.1 \mu \mathrm{m}$, intensity of $5.7 \times 10^{10} \mathrm{~W} / \mathrm{cm}^{2}$, and beam diameter of $500 \mu \mathrm{m}$. Vertical lines in the background indicate the expected wavelengths of odd harmonics. (c) Intensity scaling for fifth and seventh harmonic of few-layer graphene, showing non-perturbative scaling for both. Solid lines show theory, scaled by an arbitrary factor for each harmonic. The actual ratio predicted by theory is indicated by the vertical dashed line. (d) Fifth harmonic of few-layer and monolayer graphene at equal driving wavelengths of $3.1 \mu \mathrm{m}$, showing a broadened peak for the few-layer sample. Intensities are equal to those of panels (a) and (b), respectively.

by more than one order of magnitude for an ellipticity parameter of 0.5 relative to the response to linear polarization (Fig. 3). Measurements of ellipticity dependence were performed with incident pulse energy held constant. For comparison, the dashed line shows a representative calculated curve for gas phase harmonics for the same wavelength and intensity as we used in our experiment $^{31,32}$. It is striking that the decay of the harmonic intensity with ellipticity in graphene is very similar to what is expected for gas phase high harmonic generation.

While a six-fold symmetry is expected for the dependence of harmonic generation on the angle of linear polarization with respect to the crystal structure, no dependence was observed experimentally. This may reflect the high degree of rotational symmetry in the band structure in the vicinity of the Dirac points, as described in the simulations presented below.

To assist interpretation of our results, we use a semi- classical model of high harmonic generation. The polarization, $\mathbf{p}(\mathbf{K}, t)$, and valence and conduction band populations, $n_{v, c}(\mathbf{K}, t)$, in the transformed frame $\mathbf{K} \equiv \mathbf{k}-\mathbf{A}(t)$ (where $\mathbf{A}(t)$ is the vector potential) are found by solving a pair of coupled differential equations, as described in detail in Refs ${ }^{33}$ and $^{34}$. The inter- and intraband current densities, $\mathbf{j}_{\text {er }}$ and $\mathbf{j}_{\text {ra }}$, are then given by

$$
\mathbf{j}_{\mathrm{er}}=\frac{d}{d t} \int_{\mathrm{BZ}} \mathbf{p}(\mathbf{K}, t) d \mathbf{K}
$$

and

$$
\mathbf{j}_{\mathrm{ra}}=\sum_{m=v, c} \int_{\mathrm{BZ}} n_{m}(\mathbf{K}, t) v_{m}(\mathbf{k}) d \mathbf{K}
$$

where $\mathbf{v}_{m}(\mathbf{k})=\nabla_{\mathbf{k}} E_{m}(\mathbf{k})$ is the carrier velocity in each band, and the integrals are taken over the first Brillouin zone, BZ. The coherent sum of these oscillating currents can account for the generation of high harmonics. Our approach is similar to recent density-matrix calculations for the strong-field response of graphene ${ }^{14-17}$, which have primarily focused on the $\mathrm{THz}$ regime. In our calculations, we treat the case of a Fermi level at the Dirac point, since expected variations in the Fermi level do not have a significant effect on the predicted high harmonic spectra (see Appendix A). Our calculations focus on monolayer graphene, while our experiments were performed on both monolayer and multilayer graphene samples. We therefore do not consider here the effects of graphene multilayers, where the bandstructure is somewhat altered ${ }^{35,36}$.

Fig. 4a shows the average conduction band occupation for the entire Brillouin zone as a function of time, with the laser field also plotted (green line). The population transfer from valence to conduction band generally follows the pulse envelope. In addition to this smooth transfer of population, however, sharper small steps can be seen, at a frequency corresponding to half of the laser cycle. These rapid steps in population transfer contribute to the generation of harmonics and can be considered as analogous to plasma harmonics in the gas phase ${ }^{37}$. They

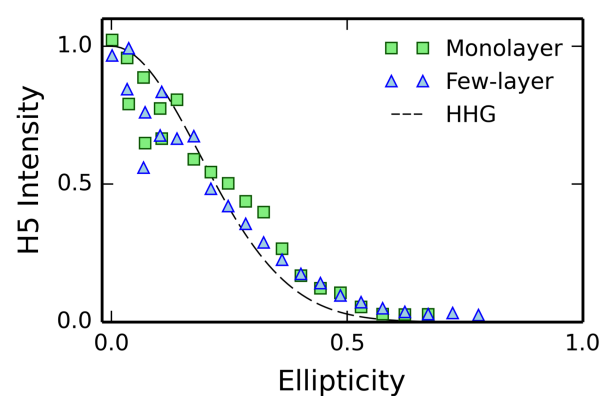

FIG. 3. Normalized fifth harmonic intensity as a function of ellipticity of incident pulse for few-layer and monolayer graphene. The dashed line shows the harmonic intensity dependence expected for high harmonic generation in Neon gas for a driving wavelength of $3.2 \mu \mathrm{m}$ and an intensity of $5 \times 10^{11} \mathrm{~W} / \mathrm{cm}^{2}$. 

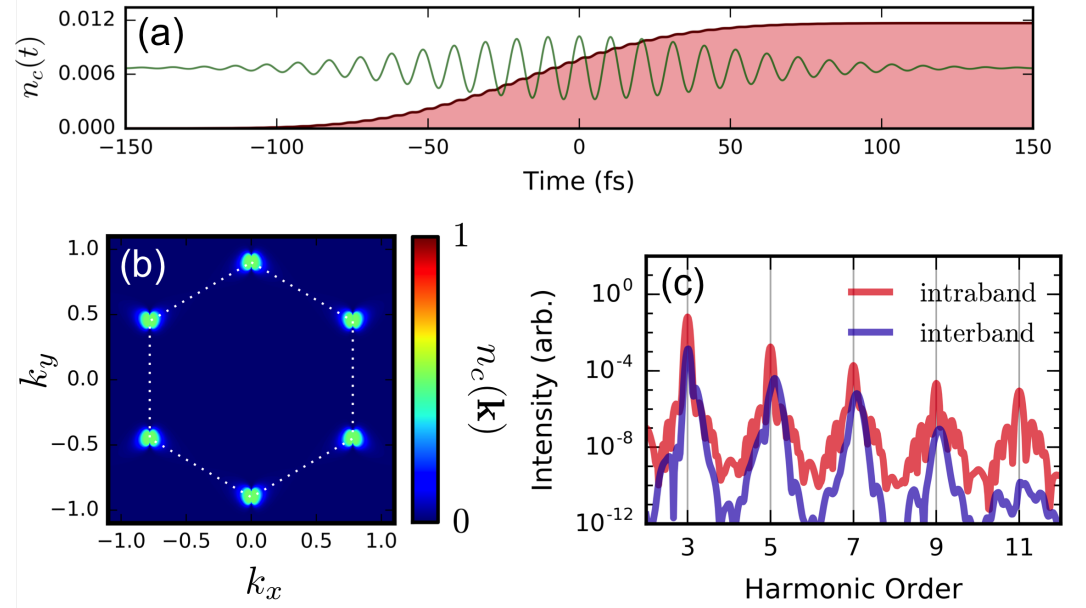
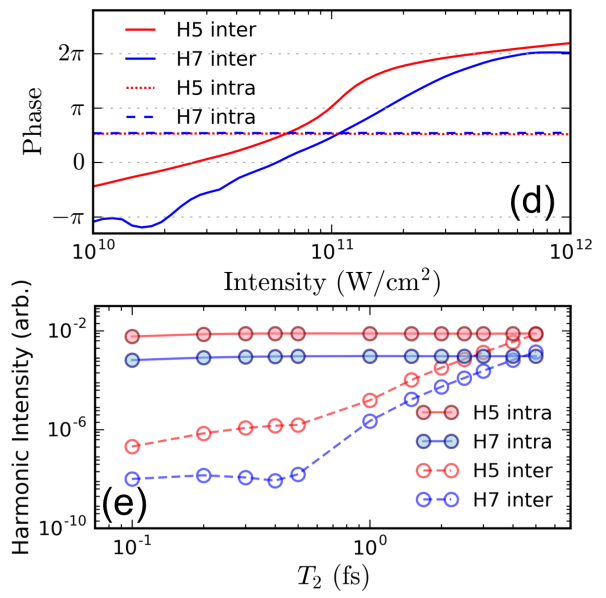

FIG. 4. Simulation of harmonic generation in single layer graphene, for a linearly polarized pulse oriented along the y-axis. (a) Total conduction band population as a function of time (red line and shaded area). As a reference, the green line shows the electric field of the optical pulse. (b) Conduction band population at the end of the pulse as function of reciprocal space coordinate. (c) Simulated harmonic spectrum with intraband (red) and interband (blue) components shown separately. $\lambda=3.1 \mu \mathrm{m}, I_{0}=10^{11} \mathrm{~W} / \mathrm{cm}^{2}$, and $T_{2}=2$ fs. (d) Phases of intraband and interband components for the fifth and seventh harmonic order as a function of incident intensity. (e) Intensities of intraband and interband components for the fifth and seventh harmonic order as a function of the dephasing time constant, $T_{2}$.

contribute to the intraband current through $n_{m}(\mathbf{K}, t)$ in Equation 2.

The conduction band population in reciprocal space at the end of the pulse (Fig. 4b) shows that population is transferred primarily at the Dirac points. Populations of electrons and holes also respond to the laser field by moving in reciprocal space, following the vector potential. This is illustrated by Video S1, in the Supplementary Material, which shows polarization and conduction band population in reciprocal space as a function of time. The field of the pulse first induces a polarization within the graphene, tightly centered at the $\mathrm{K}$ and $\mathrm{K}$ ' points, which causes a transfer of population from valence to conduction band. The resulting electron population, also centered at the $\mathrm{K}$ and $\mathrm{K}$ ' points, oscillates in the laser field, following the vector potential. The hole population in the valence band oscillates in precisely the same way, which is not shown in the video. As carriers move in reciprocal space and cross the Dirac points, their velocities rapidly switch between approximately $\pm v_{F}$, which also contributes to the intraband harmonic spectrum.

The interplay between the population dynamics and nonlinear motion in the energy bands gives rise to the simulated harmonic spectrum shown in Fig. 4c. Blue and red curves correspond to the interband and intraband contributions, respectively, as defined in Equations 1 and 2. The intraband contribution dominates throughout. However, unlike in the case of $\mathrm{THz}$ drivers ${ }^{14}$, there is a non-negligible contribution from the interband currents, which opens the possibility to observe an intensitydependent interference between interband and intraband contributions (Fig. 4d), in an analogous manner to the long and short trajectories in gas phase high harmonic generation $^{38,39}$. Furthermore the relative strength of the two contributions is found to be sensitively dependent upon the dephasing time, $T_{2}$, which is expected to be on the order of one or a few femtoseconds (see Fig. 4e). This shows that high harmonic experiments in the midinfrared open new opportunities to access ultrafast and strong-field physics of graphene.

\section{CONCLUSION}

In conclusion, we have experimentally observed nonperturbative harmonic generation from monolayer and few-layer graphene up to the fifth and seventh order, respectively, using driving wavelengths of 3 to $4 \mu \mathrm{m}$. The dependence of harmonic emission upon incident ellipticity is similar to what is predicted for gas-phase high harmonic generation for the same wavelength light. In our experiments, harmonic generation is limited by damage at high intensities. The damage threshold of graphene can be increased by using a suitable substrate, such as hexagonal boron nitride ${ }^{40}$. This suggests a way to extend harmonic generation to higher orders in future experiments, which will allow new probes of the generation mechanism $^{6}$ and of electronic structure ${ }^{3,41}$.

Non-perturbative harmonic generation in graphene from mid-infared pulsed light opens new possibilities for studying, and ultimately controlling, the dynamics of massless Dirac fermions in the strong-field regime. Based on our results, we expect an experimentally observable coherent interplay between interband and intraband gen- 
eration mechanisms. The coherent control of carrier populations in graphene's unique band structure holds promise for ultra-high speed electronics ${ }^{42}$ and optoelectronics ${ }^{43,44}$. Finally, we note that non-perturbative harmonic generation in a single atomic layer also suggests the possibility of applying high harmonic generation to the field of surface science.

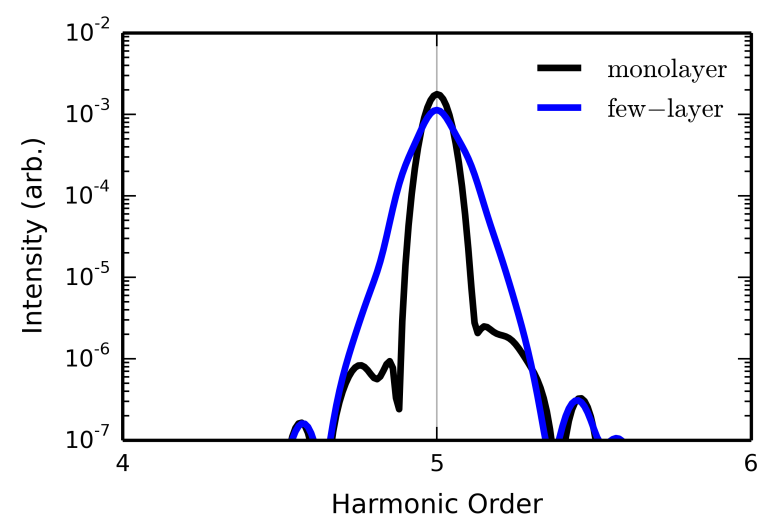

FIG. 5. Simulated fifth harmonic spectrum in the absence of self-phase modulation, representing a graphene monolayer (black), and including self-phase modulation, representing few-layer graphene (blue). A few-layer graphene thickness of $5 \mathrm{~nm}$ of graphene and a nonlinear index of $n_{2}=-0.5 \times$ $10^{-9} \mathrm{~cm}^{2} / \mathrm{W}$ are assumed.

\section{Appendix A: Spectral Broadening and Fermi Level Variation}

In order to understand the effect of self-phase modulation on the harmonics generated in few-layer graphene, we consider the propagation of the fundamental in $5 \mathrm{~nm}$ of graphene, at an intensity of $10^{11} \mathrm{~W} / \mathrm{cm}^{2}$. The simulation is the same as the one presented in the main text, except that the fundamental field is distorted by its propagation in the graphene. We found that a nonlinear index of $n_{2}=-0.5 \times 10^{-9} \mathrm{~cm}^{2} / \mathrm{W}$ reproduces the spectral broadening in the fifth harmonic of about a factor of 2 observed in experiments, as shown in Fig. 5. The spectral broadening is independent of the sign of $n_{2}$, however, recent measurements (e.g. Ref. 31) show that it is negative.

To address the role of variations in the Fermi level caused by unwanted charge traps in the substrate, we calculated the spectrum for two different Fermi energies, $0 \mathrm{eV}$ and $500 \mathrm{eV}$, illustrated in Figures $6 \mathrm{a}$ and b, respectively. The resulting spectra, shown in Fig 6c, are not significantly different, which indicates that charge-trapinduced variations of the Fermi level do not significantly affect the interpretation of our results.

\section{Appendix B: Computational Details}

For numerical simulations, we used a quantum mechanical two-band model in which polarization and band populations are coupled through the differential equations,

$$
\begin{aligned}
\dot{\pi}(\mathbf{K}, t) & =-\frac{\pi(\mathbf{K}, t)}{T_{2}}-i \Omega(\mathbf{K}, t) w(\mathbf{K}, t) e^{-i S(\mathbf{K}, t)} \\
\dot{n}_{m}(\mathbf{K}, t) & =i s_{m} \Omega^{*}(\mathbf{K}, t) \pi(\mathbf{K}, t) e^{i S(\mathbf{K}, t)}+\text { c.c. },
\end{aligned}
$$

where $m \in\{c, v\}$ labels the conduction band or valence band, respectively; $\Omega=\mathbf{d}(\mathbf{k}) \cdot \mathbf{F}(t)$ is the Rabi frequency, with $\mathbf{d}$ the transition dipole moment and $\mathbf{F}$ the electric field strength; $w \equiv n_{v}-n_{c}$ is the population difference between the two bands; $s_{m}$ is -1 or +1 for the valence and conduction band, respectively; $S$ is the classical action; and $\pi$ determines the polarization, $\mathbf{p}$, through

$$
\mathbf{p}(\mathbf{K}, t)=\mathbf{d}(\mathbf{k}) \pi(\mathbf{K}, t) e^{i S(\mathbf{K}, t)}+\text { c.c. . }
$$

This model is described in detail by Vampa et al. in Refs. 27 and 28 of the main text.

We treat a two-dimensional reciprocal space in the vector potential frame. A single reciprocal space unit cell, containing one $\mathrm{K}$ point and one $\mathrm{K}$ ' point, is divided into a finite number of polygons of unequal areas. The vertices of the polygons are defined using the equation

$$
\mathbf{K}_{i j}=\mathbf{K}_{\mathrm{K}, \mathrm{K}^{\prime}} \mp\left(\kappa_{i j} \sin \left(\theta_{j}+\Phi\right), \kappa_{i j} \cos \left(\theta_{j}+\Phi\right)\right),
$$

where

$$
\theta_{j}=\frac{\pi}{3}\left(\frac{2 j}{N_{\theta}}-1\right)
$$
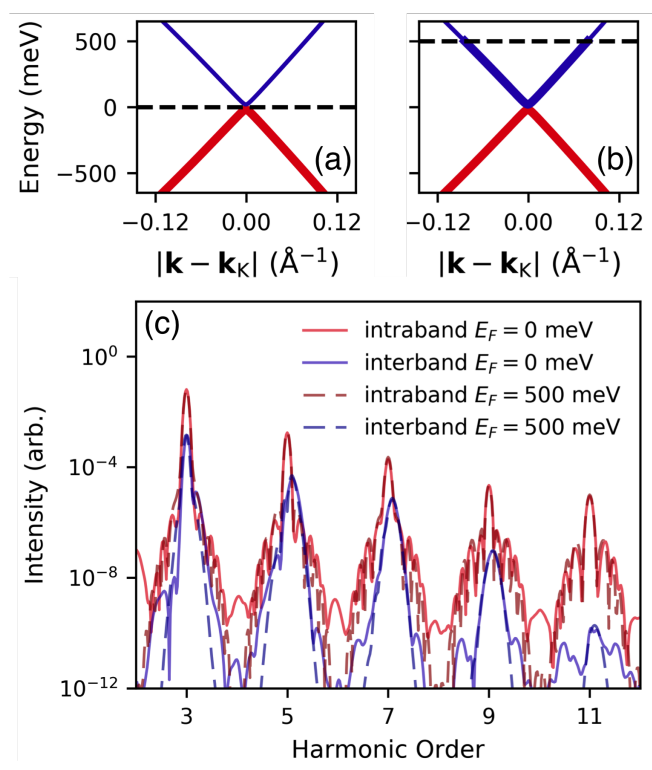

FIG. 6. Effect of Fermi level on high harmonic spectrum. (a-b) Valence and conduction bands near a Dirac point, illustrating the filling of levels up to a Fermi level of $0 \mathrm{eV}$ and $500 \mathrm{eV}$, respectively. (c) Calculated interband and intraband spectra for the Fermi levels shown in (a) and (b). 


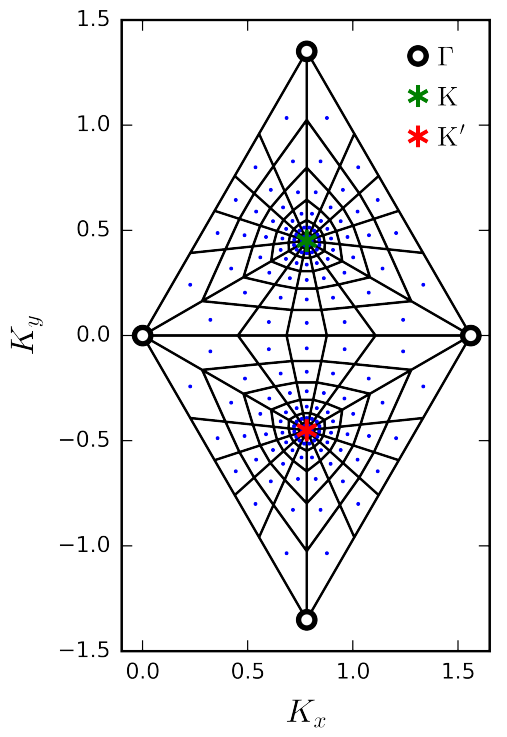

FIG. 7. Example of a grid determined by Eqn. B4, for $N_{K}=$ 8 and $N_{\theta}=6$. Black lines show polygons which define the elements of area, and blue points show their centroids, where the differential equations are evaluated.

and

$$
\kappa_{i j}=\frac{2 \pi i^{2}}{3 a N_{K}^{2}}\left[1+\frac{\left(\sec \theta_{j}-1\right) i^{2}}{N_{K}^{2}}\right] .
$$

The alternate signs in Eqn. B4 refer to the $\mathrm{K}$ and $\mathrm{K}^{\prime}$ points, respectively, located at $\mathbf{K}_{\mathrm{K}, \mathrm{K}^{\prime}}=$ $(2 \pi / \sqrt{3} a, \pm 2 \pi / 3 a)$. The indeces are $i \in\left\{0,1, \ldots, N_{K}\right\}$ and $j \in\left\{0,1, \ldots, N_{\theta}\right\}$. The numbers $N_{K}$ and $N_{\theta}$ determine the grid density. The angle $\Phi$ takes three values, $\Phi \in\{0,2 \pi / 3,4 \pi / 3\}$. The resulting grid is shown in Fig. 7, where polygons are shown in black. The differential equations, B1 and B2, are evaluated at the centroid of each polygon, shown as blue points in Fig. S1. For the simulations presented in the manuscript, we used $N_{K}=24$ and $N_{\theta}=27$.

Because of its vanishing bandgap, graphene exhibits a divergent transition dipole moment at the $\mathrm{K}$ and $\mathrm{K}$ ' points, as shown in Fig 1d. In order to address the computational difficulties that this divergence poses, we artificially limit the transition dipole moment by opening a fictitious gap of $10 \mathrm{meV}$, which creates an upper limit of approximately 60 atomic units. Since this gap is smaller than the important energy scales, it does not have a significant effect on our results. We establish this by checking for convergence of our simulation with respect to a shrinking gap. A smaller gap can be used, at the cost of longer computation time. We also check for convergence with respect to grid dimensions and density of time steps.
* marco.taucer@gmail.com

1 P. B. Corkum, Physical Review Letters 71, 1994 (1993).

${ }^{2}$ O. Schubert, M. Hohenleutner, F. Langer, B. Urbanek, C. Lange, U. Huttner, D. Golde, T. Meier, M. Kira, S. W. Koch, and R. Huber, Nature Photonics 8, 119 (2014).

3 T. T. Luu, M. Garg, S. Y. Kruchinin, a. Moulet, M. T. Hassan, and E. Goulielmakis, Nature 521, 498 (2015).

${ }^{4}$ Y. S. You, D. A. Reis, and S. Ghimire, Nature Physics 13, 345 (2017).

5 S. Ghimire, A. D. DiChiara, E. Sistrunk, P. Agostini, L. F. DiMauro, and D. A. Reis, Nature Physics 7, 138 (2011).

${ }^{6}$ G. Vampa, T. J. Hammond, N. Thire, B. E. Schmidt, F. Legare, C. R. McDonald, T. Brabec, and P. B. Corkum, Nature 522, 462 (2015).

7 G. Vampa, T. J. Hammond, N. Thiré, B. E. Schmidt, F. Légaré, D. D. Klug, and P. B. Corkum, (2016), arXiv:1605.06345.

${ }^{8}$ F. Langer, M. Hohenleutner, C. P. Schmid, C. Poellmann, P. Nagler, T. Korn, C. Schüller, M. S. Sherwin, U. Huttner, J. T. Steiner, S. W. Koch, M. Kira, and R. Huber, Nature 533, 225 (2016).

${ }^{9}$ H. Liu, Y. Li, Y. S. You, S. Ghimire, T. F. Heinz, and D. A. Reis, Nature Physics 13, 262 (2017).

10 E. Hendry, P. J. Hale, J. Moger, A. K. Savchenko, and S. A. Mikhailov, Physical Review Letters 105, 097401 (2010).

11 N. Kumar, J. Kumar, C. Gerstenkorn, R. Wang, H. Y.
Chiu, A. L. Smirl, and H. Zhao, Physical Review B 87, 121406 (2013).

12 A. K. Gupta, O. E. Alon, and N. Moiseyev, Physical Review B 68, 205101 (2003).

13 S. A. Mikhailov, Europhysics Letters 79, 27002 (2007).

14 K. L. Ishikawa, Physical Review B 82, 201402 (2010).

15 K. L. Ishikawa, New Journal of Physics 15, 055021 (2013).

16 I. Al-Naib, J. E. Sipe, and M. M. Dignam, Physical Review B 90, 245423 (2014).

17 I. Al-Naib, J. E. Sipe, and M. M. Dignam, New Journal of Physics 17, 113018 (2015).

18 H. K. Kelardeh, V. Apalkov, and M. I. Stockman, Physical Review B 93, 155434 (2016).

19 J. D. Cox, A. Marini, and F. J. G. de Abajo, Nature Communications 8, 14380 (2017).

20 P. Bowlan, E. Martinez-Moreno, K. Reimann, T. Elsaesser, and M. Woerner, Physical Review B 89, 041408 (2014).

21 P. Bowlan, E. Martinez-Moreno, K. Reimann, M. Woerner, and T. Elsaesser, New Journal of Physics 16, 013027 (2014).

22 L. A. Chizhova, F. Libisch, and J. Burgdörfer, Physical Review B 94, 075412 (2016).

23 A. H. Castro Neto, F. Guinea, N. M. R. Peres, K. S. Novoselov, and A. K. Geim, Reviews of Modern Physics 81, 109 (2009).

24 M. L. Sadowski, G. Martinez, M. Potemski, C. Berger, and W. A. deHeer, Physical Review Letters 97, 266405 (2006). 
25 S. Konschuh, M. Gmitra, and J. Fabian, Physical Review B 82, 245412 (2010).

${ }^{26}$ H. K. Kelardeh, V. Apalkov, and M. I. Stockman, Physical Review B 91, 045439 (2015).

27 H. Zhang, S. Virally, Q. Bao, L. Ping, S. Massar, N. Godbout, and P. Kockaert, Optics Letters 37, 1856 (2012).

${ }^{28}$ W. Chen, G. Wang, S. Qin, C. Wang, J. Fang, J. Qi, X. Zhang, L. Wang, H. Jia, and S. Chang, AIP Advances 3, 042123 (2013).

${ }^{29}$ L. Miao, Y. Jiang, S. Lu, B. Shi, C. Zhao, H. Zhang, and S. Wen, Photonics Research 3, 214 (2015).

30 E. Dremetsika, B. Dlubak, S.-P. Gorza, C. Ciret, M.-B. Martin, S. Hofmann, P. Seneor, D. Dolfi, S. Massar, P. Emplit, and P. Kockaert, Optics Letters 41, 3281 (2016).

31 H. Xu, H. Xiong, B. Zeng, W. Chu, Y. Fu, J. Yao, J. Chen, X. Liu, Y. Cheng, and Z. Xu, Optics letters 35, 472 (2010).

32 M. Möller, Y. Cheng, S. D. Khan, B. Zhao, K. Zhao, M. Chini, G. G. Paulus, and Z. Chang, Physical Review A 86, 011401 (2012).

33 G. Vampa, C. R. McDonald, G. Orlando, D. D. Klug, P. B. Corkum, and T. Brabec, Physical Review Letters 113, 073901 (2014).

34 G. Vampa, C. R. McDonald, G. Orlando, P. B. Corkum, and T. Brabec, Physical Review B 91, 064302 (2015).
35 B. Partoens and F. M. Peeters, Physical Review B 74, 075404 (2006).

36 B. Partoens and F. M. Peeters, Physical Review B 75, 193402 (2007).

37 F. Brunel, Journal of the Optical Society of America B 7, 521 (1990).

38 A. Zaïr, M. Holler, A. Guandalini, F. Schapper, J. Biegert, L. Gallmann, U. Keller, A. S. Wyatt, A. Monmayrant, I. A. Walmsley, E. Cormier, T. Auguste, J. P. Caumes, and P. Salières, Physical Review Letters 100, 143902 (2008).

39 D. C. Yost, T. R. Schibli, J. Ye, J. L. Tate, J. Hostetter, K. J. Schafer, and M. B. Gaarde, Nature Physics 5, 815 (2009)

40 C. R. Dean, A. F. Young, I. Meric, C. Lee, L. Wang, S. Sorgenfrei, K. Watanabe, T. Taniguchi, P. Kim, K. L. Shepard, and J. Hone, Nature Nanotechnology 5, 722 (2010).

41 G. Vampa, T. J. Hammond, N. Thire, B. E. Schmidt, F. Legare, C. R. McDonald, T. Brabec, D. D. Klug, and P. B. Corkum, Physical Review Letters 115, 193603 (2015).

42 F. Schwierz, Nature Nanotechnology 5, 487 (2010).

43 F. Bonaccorso, Z. Sun, T. Hasan, and A. C. Ferrari, Nature Photonics 4, 611 (2010).

${ }^{44}$ M. Liu, X. Yin, E. Ulin-Avila, B. Geng, T. Zentgraf, L. Ju, F. Wang, and X. Zhang, Nature 474, 64 (2011). 